\title{
A Novel Ferroptosis-related Lncrna Prognostic Signature for Colorectal Cancer by Bioinformatics Analysis
}

\section{Zhiyong Yang}

Huanggang Central Hospital Affiliated to Yangtze University

\section{Yujiao Zhang}

Huanggang Central Hospital Affiliated to Yangtze University

Jiping Wang

Huanggang Central Hospital Affiliated to Yangtze University

Guodong Yang ( $\square$ 912391303@qq.com )

Huanggang Central Hospital Affiliated to Yangtze University

Jing Liu

Huanggang Central Hospital Affiliated to Yangtze University

\section{Yaqi Zhang}

Huanggang Central Hospital Affiliated to Yangtze University

\section{Research Article}

Keywords: IncRNA, TCGA, ferroptosis, colorectal cancer, signature

Posted Date: March 11th, 2021

DOI: https://doi.org/10.21203/rs.3.rs-257325/v1

License: (c) (i) This work is licensed under a Creative Commons Attribution 4.0 International License. Read Full License 


\section{Abstract}

Background: Recently, extensive studies have shown that ferroptosis in cancer treatment has been increasingly confirmed. The current study aims to construct a robust ferroptosis-related IncRNAs signature prediction model of colorectal cancer (CRC) patients by bioinformatics analysis

Methods: The transcriptome data were abstracted from The Cancer Genome Atlas (TCGA). Differentially expressed IncRNAs were screened by comparing 568 CRC tissues with 44 adjacent non-CRC tissues. Univariate Cox regression, lasso regression, multivariate Cox regression were conducted to design a ferroptosis-related IncRNA signature. This signature's prognosis was verified by the log-rank test of Kaplan-Meier curve and the area under curve (AUC) of receiver operating characteristic (ROC) in train set, test set, and entire set. Furthermore, univariate and multivariate Cox regression were used to analyze its independent prognostic ability. The relationship of the ferroptosis-linked IncRNAs' expression and clinical variables was demonstrated by Wilcoxon rank-sum test and Kruskal-Wallis test. Gene set enrichment analysis (GSEA) was performed to signaling pathways it may involve.

Results: 2541 differentially expressed IncRNAs were screened, of which 439 are ferroptosis-related IncRNAs. A seven ferroptosis-related IncRNAs (AC005550.2, LINC02381, AL137782.1, C2orf27A, AC156455.1, AL354993.2, AC008760.1) prognostic signature was constructed, validated and evaluated. This model's prognosis in the high-risk group is obviously worse than that of the low-risk group in train set, test set, and entire set. The AUC of ROC predicting the three years survival in the train set, test set, and entire set was $0.796,0.715$, and 0.758 , respectively. Moreover, the designed molecular signature was found to be an independent prognostic variable. Compared to clinical variables, this signature's ROC curves demonstrated the second largest AUC value (0.737). The expression of these IncRNAs and the IncRNA signature are related to distant metastasis, Lymph-node status, clinical stage, T stage, KRAS mutation, BRAF mutation, MMR status, and perineural invasion. Finally, GSEA analysis results show that the signature is involved in six metabolism-related pathways.

Conclusion: The current study constructed, validated, and evaluated a seven ferroptosis-related IncRNA signature which can independently be used to predict the prognosis of CRC patients, and it may become a new therapeutic target.

\section{Background}

In 2018, a total of 1.8 million new patients with colorectal cancer (CRC) and 881,000 CRC-related deaths were reported. This accounted for one in 10 newly diagnosed cases of CRC and CRC-associated deaths. Hence, CRC is ranked as the third most prevalent but second leading cause of cancer-related mortality [1]. Despite the fact that recent advances in the genetic and molecular characterization of tumors, the 5-year survival rate of early CRC exceeds $90 \%$ whereas that of rate of metastatic colorectal cancer is below $14 \%$ [2]. Therefore, investigating promising prognostic signatures along with potential targets is considered as an essential phase to achieving this goal. 
Ferroptosis is a type of cell death that is characterized by high production of lipid ROS (L-ROS) as a result of inactivation of cellular glutathione (GSH)-dependent antioxidant defenses. This form of cell death is iron-dependent and differs from apoptosis, classic necrosis, ferroptosis, and other forms of cell death [3, 4]. Ferroptosis has been associated with the initiation of multiple diseases, including kidney injury, blood circulation diseases, conditions of the nervous system, and ischemia-reperfusion injury. It is therefore being investigated as a potential prognostic marker for various diseases [5]. Scholars have suggested that ferroptosis may be adaptive strategy used for eliminating cancerous cells and hence prevent cancer development in situations of infections, cellular stress, and nutrient deficiency [6]. Previous research has reported that some inducers, such as RSL3 [7], $\beta$-elemene [8], Resibufogenin [9], andrographis [10], bromelain [11], IMCA [12], talaroconvolutin A (TalaA) [13], ACADSB [14], erastin [15], dichloroacetate [16], and $B$. etnensis Raf. extract [17] suppressed the progression of CRC via inducing ferroptosis. Hence, it is essential to discover ferroptosis-linked biomarkers that can be applied as valuable early diagnostic as well as prognostic indicators for CRC.

Long non-coding RNAs (IncRNAs) is a class of non-coding RNAs with more than 200 nucleotides long that have apparently little or no protein-coding ability [18]. LncRNAs regulate critical biological functions related to growth of cells and survival, allosteric regulation of enzyme activities, chromatin modifications, and genomic imprinting [19]. Besides, a mounting number of studies have chronicled that IncRNAs affect cancer progression and predict dismal prognosis in diverse cancer types by modulating ferroptosis. For example, p53 related IncRNA (P53RRA) promotes apoptosis and ferroptosis of cancerous cells by activating the p53 pathway [20]. LncRNA GABPB1-AS1 regulates the status of oxidative stress in context of erastin-triggered ferroptosis in HepG2 hepatocellular carcinoma cells [21]. LncRNA-linc00336 suppresses ferroptosis in lung cancer tissues by acting as a competing endogenous RNA [22]. Linc00618 accelerates ferroptosis via inhabiting vincristine (VCR) and lymphoid-specific helicase (LSH) /SLC7A11 in leukemia [23]. In non-small cell lung cancer cells, LncRNA-MT1DP enriched on folate-modified liposomes promotes erastin-triggered ferroptosis by modulating the miR-365a-3p/NRF2 axis [24]. Hence, it is critical to explore the pivotal IncRNAs closely linked to ferroptosis along with prognosis in CRC.

This study is the first to propose a predictive model of IncRNA related to ferroptosis genes in tumors. Therefore, we postulated that ferroptosis-linked IncRNAs could be valuable prognostic biomarkers for CRC patients. Herein, we explored the expression of IncRNAs in CRC from The Cancer Genome Atlas (TCGA) and identified ferroptosis -associated IncRNAs with prognostic potential. We constructed and verified a seven ferroptosis-correlated IncRNA biosignature with the ability to estimate the survival prognosis of CRC patients.

\section{Methods}

\section{Data download and processing}

The transcriptome data (Cases (544): Primary Site (colon and rectum), Program (TCGA), Project (TCGACOAD and TCGA-READ); Files (612 including 568 CRC tissues and 44 non-CRC tissues): Data Category 
(Transcriptome Profiling), Workflow Type (HTSeq - FPKM)), Data Type (Gene Expression Quantification), clinical information (Files (548), Data Category (clinical), and Data Format (bcr xml)) were abstracted from The Cancer Genome Atlas (TCGA) web data resource (https://cancergenome.nih.gov/) on November 20, 2020 (Table 1). Patients with no follow-up time and follow-up time shorter than 30 days were not enrolled in the study.

\section{Identification of ferroptosis-associated IncRNAs (FRIncRNAs)}

The ferroptosis genes (259) were downloaded from the world's first database (ferroptosis regulators and markers and ferroptosis-disease associations (FerrDb)) (http://www.zhounan.org/ferrdb/). We employed the "limma" R package [25] to screen differentially expressed IncRNAs by comparing 568 CRC tissues with 44 adjacent non-CRC tissues. The included criteria are False Discovery Rate (FDR) $<0.05$ and $|\log F C|>1$. Furthermore, we identified ferroptosis-related IncRNAs by the correlation analysis between the IncRNAs expression levels and the ferroptosis genes based on the criteria of $\mathrm{P}<0.001$ and |Correlation Coefficient| $>0.3$.

\section{Development, verification, and assessment of prognostic biosignature}

We utilized the R language 4.0.1version "caret" package to randomly classify the entire data set (Additional file 1) with FRIncRNAs expression profiles into two sets (train set (Additional file 2) and test set (Additional file 3)), and conducted univariate Cox regression for FRIncRNAs in the train group $(\mathrm{P}<$ 0.05). Lasso regression analysis was utilized to minimize overfitting using the "glmnet" package $[26](P<$ 0.05). Afterward, multivariate Cox regression was employed to develop the optimal prognostic risk model and leveraged "coxph" and "direction = both" functions of the R language "survival" package [27] $(\mathrm{P}<$ 0.05). Then, the prognostic IncRNA signature's risk score constituting multiple IncRNAs was developed by summing up the product of each IncRNA with its corresponding coefficient. Additionally, the Proportional Hazards Assumption was tested in the Cox model. Similarly, on the basis of the previous training set's risk score formula, we applied it to the testing set as well as the entire set as validation.

This model was employed to explore each patient's survival prognosis by the Kaplan-Meier curve along with the log-rank test on the basis of the median of risk score, namely low-risk group and high-risk group in the train set, test set, entire set. The IncRNA signature's predictive power was explored by computing the AUC of 3 years using the ROC curve by the "survival ROC" package [28].

To further enhance the prognostic signature's credibility, we conducted a stratified survival prognostic analysis on gender, age, clinical stage, postoperative tumor status, CEA levels, perineural invasion, vascular invasion, mismatch repair (MMR) and gene mutation status (KRAS, BRAF).

\section{Independent and prognostic value of the IncRNA signature}


Multivariate Cox regression and univariate Cox regression analyses were conducted to analyze the independent and prognostic ability of the IncRNA signature in the train set (Additional file 4), test set (Additional file 5), and entire set (Additional file 6). The clinical parameters include age, gender, clinical stage, T stage, lymph nodes as well as distant metastasis. Besides, compared with clinical variables, The ROC curve was employed to explore whether the IncRNA biosignature has better predictive power. The "rms" package was employed to construct the nomogram according to the multivariate Cox regression result $(P<0.05)$. To further investigate whether the ferroptosis -associated IncRNAs are involved in CRC development, we explored the relationship of the ferroptosis-linked IncRNAs' expression with clinical variables using the Wilcoxon rank-sum test and Kruskal-Wallis test.

GSEA analysis of the IncRNA signature.

Gene set enrichment analysis (GSEA4.1.0) downloaded from https://www.gseamsigdb.org/gsea/index.jsp website was employed to identify the biological function of the prediction model [29]. Based on the median expression of IncRNA signature riskScore in 568 tumor samples, we divided them into low and high-risk groups for KEGG analysis of GSEA. The abundant signaling cascades in each phenotype were based on the normalized enrichment score (NES), the nominal (NOM) P-value as well as the false discovery rate (FDR). FDR $<25 \%$ and NOM P-value $<5 \%$ serve as a standard for inclusion.

\section{6 | Statistical Analysis}

R software 4.0.3 version and attached packages were employed to conduct data analyses. All the statistical analyses were two-sided. $\mathrm{P}<0.05$ signified of statistical significance.

\section{Results}

\section{Screening of ferroptosis-related IncRNAs in CRC}

Comparing CRC tissues with adjacent non-CRC tissues, 2541 differentially expressed IncRNAs were found, of which 1805 are up-regulated and 736 are down-regulated (Additional file 7). The correlation results between 259 ferroptosis-related genes and differentially expressed IncRNAs shown that there are 439 ferroptosis-related IncRNAs (FRIncRNAs) (Additional file 8).

\section{Construction, validation, and evaluation of a seven ferroptosis-related IncRNAs prognostic signature}

The entire set $(N=506)$ with 439 FRIncRNAs expression data was randomized into the test set $(N=252)$ and train set $(\mathrm{N}=254)$. In the univariate Cox regression assessment, 22 FRIncRNAs modulated the overall survival of the patients in the train set (Fig. 1a). Lasso regression was used for further analysis to eliminate overfitting IncRNAs, and the 16 IncRNAs we obtained were used for the subsequent multivariate Cox regression analysis (Fig. 1b-d) (concordance index [C-index], 0.75). The ferroptosis-associated IncRNA prognostic biosignature was developed based by summing up the product of each IncRNA 
expression with its corresponding coefficient in multivariate Cox regression as indicated below: IncRNA biosignature risk score $=(0.136 \times$ expression of AC005550.2) $+(0.240 \times$ expression of LINC02381 $)+$ $(-1.407 \times$ expression of AL137782.1) + (0.365x expression of C2orf27A $)+(0.235 \times$ expression of $A C 156455.1)+(0.280 \times$ expression of AL354993.2 1) + (0.653x expression of AC008760.1). Analysis using the Proportional Hazards Assumption in the Cox model revealed that all the $\mathrm{P}$ values $>0.05$, implying they conformed to the PH test (Additional file 9).

According to the median value of the risk score, results of the Kaplan-Meier curves demonstrate that the high-risk group has a remarkably dismal overall survival (OS) in contrast with the low-risk group in the train set $(P=2.899 \mathrm{E}-06)$, test set $(P=5.314 \mathrm{E}-03)$, and entire set $(P=1.1 \mathrm{E}-06)($ Fig. 2a-c). The train set shows three years' OS for patients with high and low-risk group were $60.6 \%$ and $90.5 \%$, respectively. The test set is $63.9 \%$ and $90.1 \%$, respectively. The entire set is $60.6 \%$ and $90.5 \%$, respectively. The AUC of three years dependent ROC for the seven-IncRNA biosignature achieves $0.796,0.715$, and 0.758 respectively in the train set, test set, and entire set (Fig. 2d-f), which demonstrate the good performance of the model in estimating the CRC patients' OS. The mortality rate was higher in patients with high-risk scores relative to those with low-risk scores in the three sets (Fig. 2g-i). The six InRNAs' (AC005550.2, LINC02381, C2orf27A, AC156455.1, AL354993.2, AC008760.1) expression of signature were lower in low-risk group compared to the high-risk group in cluster heat map, AL137782.1 oppositely (Fig. 2j-I).

It is worth noting that AC156455.1, and AL354993.2's high expression of this IncRNA signature also has a worse OS than low (Fig. 3). The association of the seven IncRNAs with ferroptosis genes is shown in Fig. 4. In addition, we stratified according to various clinical factors (clinical stage, gender, age, CEA levels, MMR status, postoperative tumor status, perineural invasion, vascular invasion, KRAS mutation, BRAF mutation) and applied the prognostic model to OS detection, which is shown in Fig. 5, the results shown that the signature has good predictive significance for CRC patients in most stratification factors, and part of results are not satisfactory $(P>0.05)$, which might be due to there are not enough samples in these stratification.

\section{Independent prognostic analysis of the seven ferroptosis-associated IncRNAs signature and its correlation with clinical variables.}

The Univariate Cox regression assessment demonstrated that the IncRNA biosignature risk score was evidently correlated with the patients' OS (hazard ratio $\mathrm{HR}=1.224$, confidence interval $95 \% \mathrm{Cl}=1.149$ $1.303, \mathrm{P}=3.69 \mathrm{E}-10)$ in train set, $(\mathrm{HR}=1.160,95 \% \mathrm{Cl}=1.016-1.325, \mathrm{P}=0.028)$ test set, and $(\mathrm{HR}=1.179$, $95 \% \mathrm{Cl}=1.125-1.235, \mathrm{P}=7.04 \mathrm{E}-12$ ) entire set (Table 2). Moreover, the multivariate Cox regression analysis demonstrated that the IncRNA biosignature risk score remained independent with OS considering other conventional clinical factors including Lymph-node status, the clinical stage, distant metastasis, and $\mathrm{T}$ stage $(\mathrm{HR}=1.187,95 \% \mathrm{Cl}=1.107-1.273, \mathrm{P}=1.39 \mathrm{E}-06)$ in the train set, and $(\mathrm{HR}=1.122$, $95 \% \mathrm{Cl}=1.067-1.180, \mathrm{P}=8.33 \mathrm{E}-06)$ in entire set, in spite of $\mathrm{P}>0.05(\mathrm{HR}=1.059,95 \% \mathrm{Cl}=0.995-1.289, \mathrm{P}$ $=0.059)$ in test set. Meanwhile, $T$ stage and age were demonstrated as an independent prognostic index. 
Compared to clinical variables, this signature risk score's ROC curves of three years demonstrate the second-largest AUC value (0.737) (Fig. 6).

Based on the stratification of clinical variables, the correlation between the IncRNAs and clinical variables shows that LINC02381' expression is related to T stage, Lymph-node status, and clinical stage, KRAS mutation, BRAF mutation, and perineural invasion. C2orf27A' expression is associated with T stage, Lymph-node status, clinical stage, KRAS mutation, MMR. AC156455.1' expression is correlated to Lymphnode status. AL354993.2' expression is connected to distant metastasis, Lymph-node status, clinical stage, KRAS mutation. AC008760.1' expression is concerning to Lymph-node status, distant metastasis, clinical stage, KRAS mutation. AL137782.1' expression is linked to KRAS mutation. The IncRNA signature' riskscore is coupled to T stage, Lymph-node status, distant metastasis, clinical stage, and KRAS mutation. (Fig. 7).

\section{Functional enrichment analysis of the seven ferroptosis-related IncRNAs signature.}

GSEA analysis is used to discover potential biological functions of the seven ferroptosis-associated IncRNAs signature of CRC (Fig. 8). The results showed that three signaling pathways (KEGG_HEDGEHOG_SIGNALING_PATHWAY, KEGG_ARACHIDONIC_ACID_METABOLISM, KEGG_ALPHA_LINOLENIC_ACID_METABOLISM) are obviously enriched in the high-risk group, and three signaling cascades (KEGG_FRUCTOSE_AND_MANNOSE_METABOLISM, KEGG_PENTOSE_PHOSPHATE_PATHWAY KEGG_CITRATE_CYCLE_TCA_CYCLE) were abundant in the low-risk group by c2.cp.kegg.v7.2.symbols.gmt. These results suggest that this signature model may influence $\mathrm{CRC}$ progression and prognosis mainly through metabolism-related pathways

\section{Discussion}

$\mathrm{CRC}$ is a common and aggressive cancer with poor survival and prognosis, mainly due to the prone to metastasis to the liver and lung [30]. Given that there are no accurate and sensitive markers to predict the prognosis of CRC patients, it is crucial to investigate and develop more specific biomarkers to improve the survival of patients. Although the current treatment methods have made great advancements, the prognosis is still very poor. Ferroptosis is differs from other types of cell death in terms of biochemically and morphologically and has been shown to regulate cancer development [3]. More and more reports have documented that IncRNA plays a very important role in regulating gene expression and regulation in tumor $[19,31]$. In addition, many IncRNAs influence the progression of CRC by regulating ferroptosis. However, there are no reports on that prognostic model of IncRNA related to ferroptosis was constructed. Although two previous genetic prognostic models of ferroptosis have been reported in hepatocellular carcinoma [32] and glioma [33], our study is the first to report the study of ferroptosis-related IncRNA prognostic models in CRC.

In the present study, we downloaded ferroptosis genes from FerrDb, and used the R language and its attached packages to find differentially expressed IncRNAs related to ferroptosis (FRIncRNAs). We randomly grouped all the patients into train set as well as the test set, then a seven ferroptosis-related 
IncRNAs signature model (AC005550.2, LINC02381, AL137782.1, C2orf27A, AC156455.1, AL354993.2, AC008760.1) was established through univariate Cox regression, Lasso regression, as well as multivariate Cox regression in the train set. At the same time, the biosignature was verified in the test set as well as the entire set. On the basis of the median risk score, the Kaplan-Meier curves revealed that the high-risk group had an evidently dismal overall survival relative to the low-risk group in the three data sets and various clinical stratification factors. Assessment of the biosignature for OS in the three sets by ROC curve exhibited well predictive value. The Univariate Cox regression as well as the multivariate Cox regression analyses demonstrated that the biosignature had independent prognostic ability considering other conventional clinical variables for CRC patients. On the basis of the multivariate Cox regression results, we developed the nomogram of the clinical prediction model. Furthermore, the seven IncRNAs and the signature model are linked to the T stage, Lymph-node status, distant metastasis, clinical stage, KRAS mutation, BRAF mutation, and perineural invasion to varying degrees. Finally, GSEA analysis results show that the signature model is involved in six KEGG signal pathways based on high and low-risk group, such as KEGG_HEDGEHOG_SIGNALING_PATHWAY, KEGG_ARACHIDONIC_ACID_METABOLISM, KEGG_ALPHA_LINOLENIC_ACID_METABOLISM, KEGG_FRUCTOSE_AND_MANNOSE_METABOLISM, KEGG_PENTOSE_PHOSPHATE_PATHWAY, and KEGG_CITRATE_CYCLE_TCA_CYCLE. These results suggest that this signature model may influence CRC progression and prognosis mainly through metabolism-related pathways

Before this study, many prognostic models of CRC have been constructed from different research perspectives. For example, using bioinformatics tool, Yang $\mathrm{G}$ et al. identified a five-microRNA signature which could predict the prognosis of CRC [34]. Bai $\mathrm{J}$ et al. developed a novel 14-gene immune-related signature which showed good performance in prognosis prediction of CRC [35]. Zhang $\mathrm{J}$ et al. screened gene signature of prognostic m6A modulators in CRC [36]. Using immune and inflammatory cells, Xiao G et al. constructed a risk signature for assessing efficiency of chemotherapy and distant metastases of $\mathrm{CRC}$ [37]. Tokunaga $\mathrm{R}$ et al. presented a 12-chemokine signature for evaluating tumor recurrence in CRC [38]. Zong $Z$ et al. demonstrated a prognostic alternative splicing signature by differential splicing patterns of 13 genes in CRC [39]. Li K et al. revealed a six gene-specific DNA methylation signature for CRC [40]. Zhou Z et al. developed and validated of an autophagy signature based on 5 autophagy genes which could evaluate the survival of CRC patients after surgery [41]. Wu B et al. developed an immune infiltration-related eight-gene prognostic signature in CRC microenvironment [42]. These examples are only the tip of the iceberg. At present, there are no reports about the prognostic model of IncRNA related to ferroptosis in tumors.

Among these IncRNAs of the signature, some studies have shown that LINC02381 is related to immune gene [43] and autophagy gene [44] in colon adenocarcinoma. Interestingly, our research shows that this IncRNA is also related to ferroptosis, which is worthy of our in-depth thinking. In addition, Jafarzadeh, M et al' study revealed that LINC02381 might suppress human CRC tumorigenesis partly by regulating PI3K signaling pathway [45]. Meanwhile, LINC02381 inhibits gastric cancer progression and metastasis through regulating wnt signaling pathway [46]. However, LINC02381 functions as a cancer-promoting gene to promote cell migration and viability by regulating mir-133b / RhoA in cervical cancer [47]. 
AC008760.1 was reported to be related to autophagy, and Li et al. constructed a autophagy-related IncRNA prognosis model in CRC [48]. The remaining IncRNAs have not seen relevant reports in previous studies, which are worthy of further research.

Our study found that the expression of these IncRNAs and the constructed prognostic signature were closely related to the patient's clinical stage, distant metastasis, Lymph-node status, T stage, MMR status, BRAF mutation, KRAS mutation, and perineural invasion, especially the MMR status, BRAF mutation and KRAS mutation. These features have an important guiding significance for patients' medication. So can we explore whether these IncRNAs regulate these variables and how to regulate them? There have been many studies about ferroptosis in the drug resistance of tumor patients $[49,50]$. The current study demonstrated the prognostic significance of these ferroptosis-related IncRNAs and signature in CRC. Therefore, we have reason to believe that these IncRNAs are worthy of in-depth research in tumor resistance mechanisms.

Our current study also has some limitations. First, we use the data in the TCGA database as the starting point for research; although the model has been internally verified, it is still needed for further verification in external data; second, TCGA's race is mainly white (75\%), and whether the model fits other race needs further verification. Third, the analysis of the IncRNA expression of the model and the KEGG function enrichment analysis by the GSEA model requires further cell function experimental analysis.

\section{Conclusion}

Herein, we established a novel ferroptosis-related IncRNA prognostic signature model comprising seven IncRNAs (AC005550.2, LINC02381, AL137782.1, C2orf27A, AC156455.1, AL354993.2, AC008760.1) in CRC. In the future, the seven ferroptosis-related IncRNA prognostic biosignature could enhance predictive accuracy as well as guide individualized therapy for CRC patients with prospective validation.

\section{Abbreviations}

CRC: Colorectal cancer; GSEA: Gene set enrichment analysis; FDR: False discovery rate; OS: Overall survival; HRs: Hazard ratios; Cl: Confidence interval; TCGA: The Cancer Genome Atlas; AUC: Area under curve; ROC: Receiver operating characteristic; KEGG: Kyoto Encyclopedia of Genes and Genomes.

\section{Declarations}

\section{Ethics approval and consent to participate}

LncRNA and mRNA sequencing profiles were obtained from the TCGA data portal, which is a publicly available dataset. Therefore, no ethics approval is needed.

\section{Consent for publication}


Not applicable

\section{Availability of data and materials}

The transcriptome data (HTSeq - FPKM)) and clinical information were downloaded from The Cancer Genome Atlas (TCGA) (https://cancergenome.nih.gov/). The ferroptosis genes were downloaded from Human Ferroptosis Database (HADb) (http://www.ferroptosis.lu).

\section{Competing interests}

The authors declare that they have no competing interests.

\section{Funding}

The present study was supported by the Huanggang Municipal General Project of China (grant no. KJS0012).

\section{Authors' contributions}

Yujiao Zhang downloaded the IncRNA and mRNA expression information. Zhiyong Yang constructed the IncRNA signature model and performed the statistical analysis using $\mathrm{R}$ language software, and wrote the first draft of the manuscript. Jiping Wang revised the manuscript. Guodong Yang and Jing Liu contributed conception and design of the study and checked the manuscript.

\section{Acknowledgements}

Not applicable.

\section{References}

1. Bray F, Ferlay J, Soerjomataram I, Siegel RL, Torre LA, Jemal A: Global cancer statistics 2018: GLOBOCAN estimates of incidence and mortality worldwide for 36 cancers in 185 countries. $C A$ Cancer J Clin 2018, 68(6):394-424.

2. Ferlay J, Steliarova-Foucher E, Lortet-Tieulent J, Rosso S, Coebergh JW, Comber H, Forman D, Bray F: Cancer incidence and mortality patterns in Europe: estimates for 40 countries in 2012. Eur J Cancer 2013, 49(6):1374-1403.

3. Dixon SJ, Lemberg KM, Lamprecht MR, Skouta R, Zaitsev EM, Gleason CE, Patel DN, Bauer AJ, Cantley AM, Yang WS et al: Ferroptosis: an iron-dependent form of nonapoptotic cell death. Cell 2012, 149(5):1060-1072.

4. Yang WS, SriRamaratnam R, Welsch ME, Shimada K, Skouta R, Viswanathan VS, Cheah JH, Clemons PA, Shamji AF, Clish CB et al: Regulation of ferroptotic cancer cell death by GPX4. Cell 2014, 156(12):317-331. 
5. Li J, Cao F, Yin HL, Huang ZJ, Lin ZT, Mao N, Sun B, Wang G: Ferroptosis: past, present and future. Cell Death Dis 2020, 11(2):88.

6. Fearnhead HO, Vandenabeele $\mathrm{P}$, Vanden Berghe $\mathrm{T}$ : How do we fit ferroptosis in the family of regulated cell death? Cell Death Differ 2017, 24(12):1991-1998.

7. Sui X, Zhang R, Liu S, Duan T, Zhai L, Zhang M, Han X, Xiang Y, Huang X, Lin H et al: RSL3 Drives Ferroptosis Through GPX4 Inactivation and ROS Production in Colorectal Cancer. Front Pharmacol 2018, 9:1371.

8. Chen P, Li X, Zhang R, Liu S, Xiang Y, Zhang M, Chen X, Pan T, Yan L, Feng J et al: Combinative treatment of beta-elemene and cetuximab is sensitive to KRAS mutant colorectal cancer cells by inducing ferroptosis and inhibiting epithelial-mesenchymal transformation. Theranostics 2020, 10(11):5107-5119.

9. Shen LD, Qi WH, Bai JJ, Zuo CY, Bai DL, Gao WD, Zong XL, Hao TT, Ma Y, Cao GC: Resibufogenin inhibited colorectal cancer cell growth and tumorigenesis through triggering ferroptosis and ROS production mediated by GPX4 inactivation. Anat Rec (Hoboken) 2020.

10. Sharma P, Shimura T, Banwait JK, Goel A: Andrographis-mediated chemosensitization through activation of ferroptosis and suppression of beta-catenin/Wnt-signaling pathways in colorectal cancer. Carcinogenesis 2020, 41(10):1385-1394.

11. Park S, Oh J, Kim M, Jin EJ: Bromelain effectively suppresses Kras-mutant colorectal cancer by stimulating ferroptosis. Anim Cells Syst (Seoul) 2018, 22(5):334-340.

12. Zhang $L$, Liu W, Liu F, Wang Q, Song $M, Y u$ Q, Tang $K$, Teng $T$, Wu D, Wang $X$ et al: IMCA Induces Ferroptosis Mediated by SLC7A11 through the AMPK/mTOR Pathway in Colorectal Cancer. Oxid Med Cell Longev 2020, 2020:1675613.

13. Xia Y, Liu S, Li C, Ai Z, Shen W, Ren W, Yang X: Discovery of a novel ferroptosis inducertalaroconvolutin A-killing colorectal cancer cells in vitro and in vivo. Cell Death Dis 2020, 11(11):988.

14. Lu D, Yang Z, Xia Q, Gao S, Sun S, Luo X, Li Z, Zhang X, Li X: ACADSB regulates ferroptosis and affects the migration, invasion, and proliferation of colorectal cancer cells. Cell Biol Int 2020, 44(11):2334-2343.

15. Xu X, Zhang X, Wei C, Zheng D, Lu X, Yang Y, Luo A, Zhang K, Duan X, Wang Y: Targeting SLC7A11 specifically suppresses the progression of colorectal cancer stem cells via inducing ferroptosis. Eur $J$ Pharm Sci 2020, 152:105450.

16. Sun J, Cheng X, Pan S, Wang L, Dou W, Liu J, Shi X: Dichloroacetate attenuates the stemness of colorectal cancer cells via trigerring ferroptosis through sequestering iron in lysosomes. Environ Toxicol 2020.

17. Malfa GA, Tomasello B, Acquaviva R, Genovese C, La Mantia A, Cammarata FP, Ragusa M, Renis M, Di Giacomo C: Betula etnensis Raf. (Betulaceae) Extract Induced H0-1 Expression and Ferroptosis Cell Death in Human Colon Cancer Cells. Int J Mol Sci 2019, 20(11).

18. Mercer TR, Dinger ME, Mattick JS: Long non-coding RNAs: insights into functions. Nature reviews Genetics 2009, 10(3):155-159. 
19. Quinn JJ, Chang HY: Unique features of long non-coding RNA biogenesis and function. Nat Rev Genet 2016, 17(1):47-62.

20. Mao C, Wang X, Liu Y, Wang M, Yan B, Jiang Y, Shi Y, Shen Y, Liu X, Lai W et al: A G3BP1-Interacting IncRNA Promotes Ferroptosis and Apoptosis in Cancer via Nuclear Sequestration of p53. Cancer Res 2018, 78(13):3484-3496.

21. Qi W, Li Z, Xia L, Dai J, Zhang Q, Wu C, Xu S: LncRNA GABPB1-AS1 and GABPB1 regulate oxidative stress during erastin-induced ferroptosis in HepG2 hepatocellular carcinoma cells. Sci Rep 2019, 9(1):16185.

22. Wang M, Mao C, Ouyang L, Liu Y, Lai W, Liu N, Shi Y, Chen L, Xiao D, Yu F et al: Long noncoding RNA LINC00336 inhibits ferroptosis in lung cancer by functioning as a competing endogenous RNA. Cell Death Differ 2019, 26(11):2329-2343.

23. Wang Z, Chen X, Liu N, Shi Y, Liu Y, Ouyang L, Tam S, Xiao D, Liu S, Wen F et al: A Nuclear Long NonCoding RNA LINC00618 Accelerates Ferroptosis in a Manner Dependent upon Apoptosis. Mol Ther 2020.

24. Gai C, Liu C, Wu X, Yu M, Zheng J, Zhang W, Lv S, Li W: MT1DP loaded by folate-modified liposomes sensitizes erastin-induced ferroptosis via regulating miR-365a-3p/NRF2 axis in non-small cell lung cancer cells. Cell Death Dis 2020, 11(9):751.

25. Ritchie ME, Phipson B, Wu D, Hu Y, Law CW, Shi W, Smyth GK: limma powers differential expression analyses for RNA-sequencing and microarray studies. Nucleic Acids Res 2015, 43(7):e47.

26. Simon N, Friedman J, Hastie T, Tibshirani R: Regularization Paths for Cox's Proportional Hazards Model via Coordinate Descent. J Stat Softw 2011, 39(5):1-13.

27. Groeneveld CS, Chagas VS, Jones SJM, Robertson AG, Ponder BAJ, Meyer KB, Castro MAA: RTNsurvival: an R/Bioconductor package for regulatory network survival analysis. Bioinformatics 2019, 35(21):4488-4489.

28. Heagerty PJ, Lumley T, Pepe MS: Time-dependent ROC curves for censored survival data and a diagnostic marker. Biometrics 2000, 56(2):337-344.

29. Subramanian A, Tamayo P, Mootha VK, Mukherjee S, Ebert BL, Gillette MA, Paulovich A, Pomeroy SL, Golub TR, Lander ES et al: Gene set enrichment analysis: a knowledge-based approach for interpreting genome-wide expression profiles. Proc Natl Acad Sci U S A 2005, 102(43):15545-15550.

30. Adam R, de Gramont A, Figueras J, Kokudo N, Kunstlinger F, Loyer E, Poston G, Rougier P, RubbiaBrandt L, Sobrero A et al: Managing synchronous liver metastases from colorectal cancer: a multidisciplinary international consensus. Cancer Treat Rev 2015, 41(9):729-741.

31. Bhan A, Soleimani M, Mandal SS: Long Noncoding RNA and Cancer. A New Paradigm. Cancer Res 2017, 77(15):3965-3981.

32. Liang JY, Wang DS, Lin HC, Chen XX, Yang H, Zheng Y, Li YH: A Novel Ferroptosis-related Gene Signature for Overall Survival Prediction in Patients with Hepatocellular Carcinoma. Int J Bio/ Sci 2020, 16(13):2430-2441. 
33. Zhuo S, Chen Z, Yang Y, Zhang J, Tang J, Yang K: Clinical and Biological Significances of a Ferroptosis-Related Gene Signature in Glioma. Front Oncol 2020, 10:590861.

34. Yang G, Zhang Y, Yang J: A Five-microRNA Signature as Prognostic Biomarker in Colorectal Cancer by Bioinformatics Analysis. Front Oncol 2019, 9:1207.

35. Bai J, Zhang $X$, Xiang ZX, Zhong PY, Xiong B: Identification of prognostic immune-related signature predicting the overall survival for colorectal cancer. Eur Rev Med Pharmacol Sci 2020, 24(3):11341141.

36. Zhang J, Cheng X, Wang J, Huang Y, Yuan J, Guo D: Gene signature and prognostic merit of M6a regulators in colorectal cancer. Exp Biol Med (Maywood) 2020, 245(15):1344-1354.

37. Hu X, Li YQ, Ma XJ, Zhang L, Cai SJ, Peng JJ: A Risk Signature With Inflammatory and T Immune Cells Infiltration in Colorectal Cancer Predicting Distant Metastases and Efficiency of Chemotherapy. Front Oncol 2019, 9:704.

38. Tokunaga R, Nakagawa S, Sakamoto Y, Nakamura K, Naseem M, Izumi D, Kosumi K, Taki K, Higashi T, Miyata $T$ et al: 12-Chemokine signature, a predictor of tumor recurrence in colorectal cancer. Int $J$ Cancer 2020, 147(2):532-541.

39. Zong Z, Li H, Yi C, Ying H, Zhu Z, Wang H: Genome-Wide Profiling of Prognostic Alternative Splicing Signature in Colorectal Cancer. Front Oncol 2018, 8:537.

40. Li K, Zeng L, Wei H, Hu J, Jiao L, Zhang J, Xiong Y: Identification of gene-specific DNA methylation signature for Colorectal Cancer. Cancer Genet 2018, 228-229:5-11.

41. Zhou Z, Mo S, Dai W, Ying Z, Zhang L, Xiang W, Han L, Wang Z, Li Q, Wang R et al: Development and Validation of an Autophagy Score Signature for the Prediction of Post-operative Survival in Colorectal Cancer. Front Oncol 2019, 9:878.

42. Wu B, Tao L, Yang D, Li W, Xu H, He Q: Development of an Immune Infiltration-Related Eight-Gene Prognostic Signature in Colorectal Cancer Microenvironment. Biomed Res Int 2020, 2020:2719739.

43. Li Z, Wang D, Yin H: A seven immune-related IncRNA signature predicts the survival of patients with colon adenocarcinoma. Am J Transl Res 2020, 12(11):7060-7078.

44. Zhou W, Zhang S, Li HB, Cai Z, Tang S, Chen LX, Lang JY, Chen Z, Chen XL: Development of Prognostic Indicator Based on Autophagy-Related IncRNA Analysis in Colon Adenocarcinoma. Biomed Res Int 2020, 2020:9807918.

45. Jafarzadeh M, Soltani BM, Soleimani M, Hosseinkhani S: Epigenetically silenced LINC02381 functions as a tumor suppressor by regulating PI3K-Akt signaling pathway. Biochimie 2020, 171172:63-71.

46. Jafarzadeh M, Soltani BM: Long Noncoding RNA LOC400043 (LINC02381) Inhibits Gastric Cancer Progression Through Regulating Wnt Signaling Pathway. Front Oncol 2020, 10:562253.

47. Chen X, Zhang Z, Ma Y, Su H, Xie P, Ran J: LINC02381 Promoted Cell Viability and Migration via Targeting miR-133b in Cervical Cancer Cells. Cancer Manag Res 2020, 12:3971-3979. 
48. Wei J, Ge X, Tang Y, Qian Y, Lu W, Jiang K, Fang Y, Hwang M, Fu D, Xiao Q et al: An AutophagyRelated Long Noncoding RNA Signature Contributes to Poor Prognosis in Colorectal Cancer. J Oncol 2020, 2020:4728947.

49. Friedmann Angeli JP, Krysko DV, Conrad M: Ferroptosis at the crossroads of cancer-acquired drug resistance and immune evasion. Nat Rev Cancer 2019, 19(7):405-414.

50. Elgendy SM, Alyammahi SK, Alhamad DW, Abdin SM, Omar HA: Ferroptosis: An emerging approach for targeting cancer stem cells and drug resistance. Crit Rev Oncol Hematol 2020, 155:103095.

\section{Tables}

Due to technical limitations, table 1 and 2 is only available as a download in the Supplemental Files section.

\section{Figures}

a
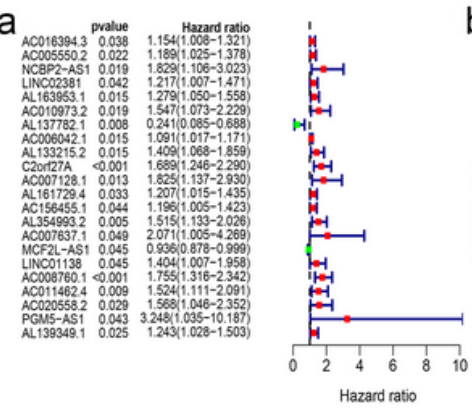

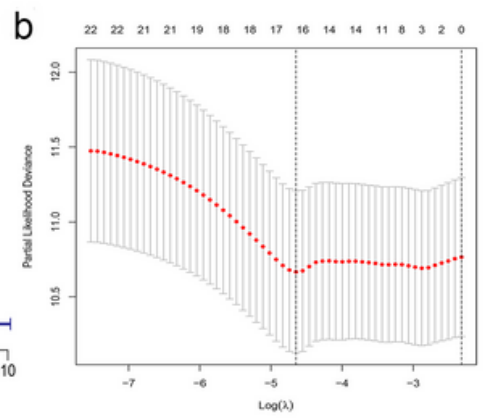

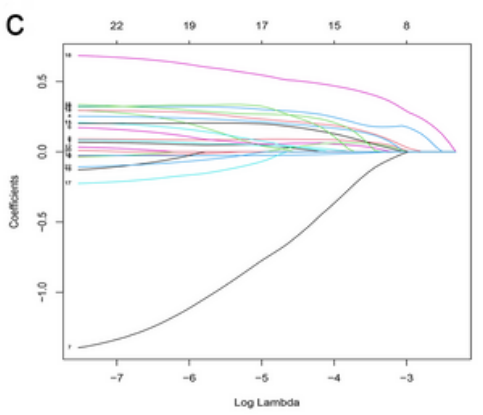

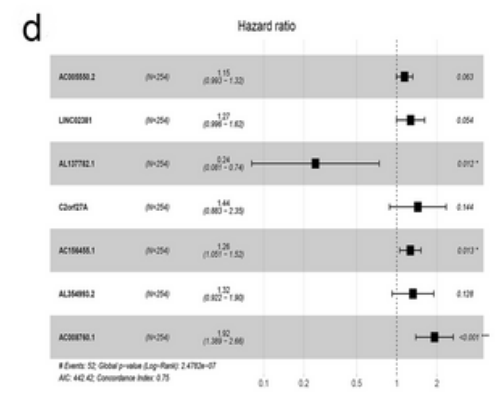

Figure 1

Construction of the ferroptosis-related IncRNAs prognostic signature. a Univariate Cox regression. b-c Lasso regression. d Multivariate Cox regression. 
Train set

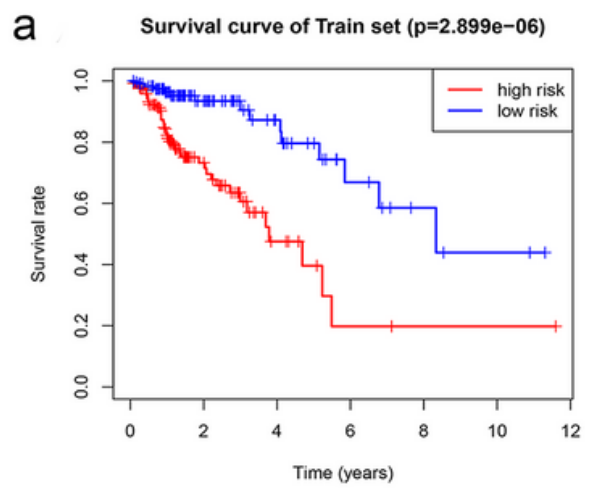

d
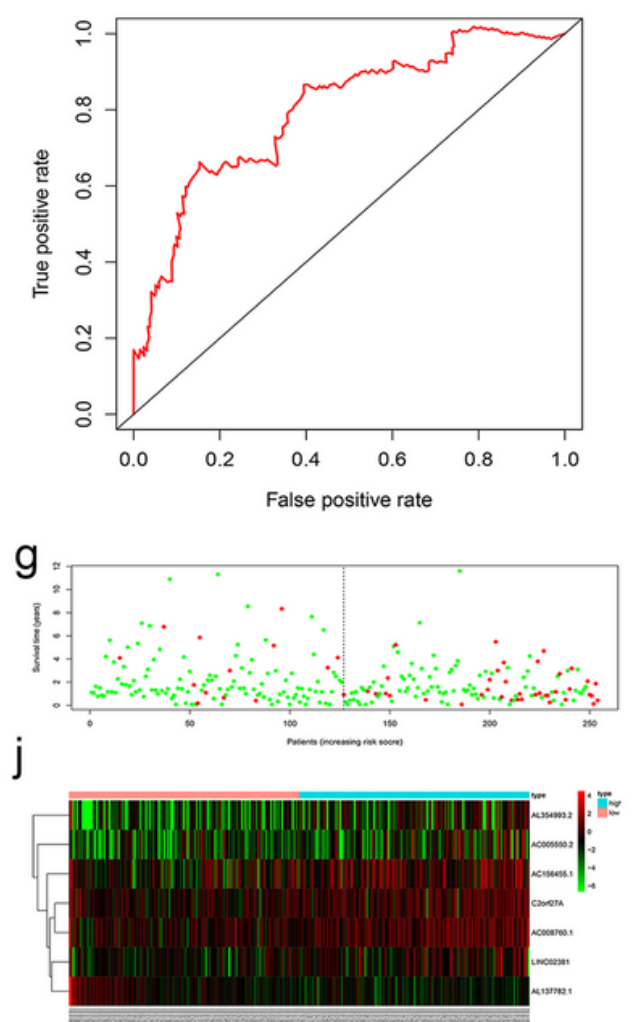

Test set

b

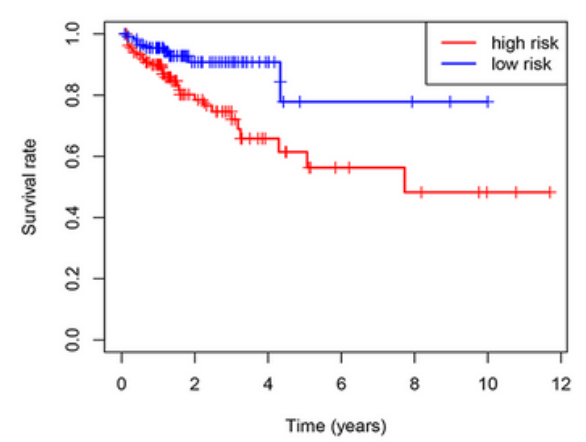

e

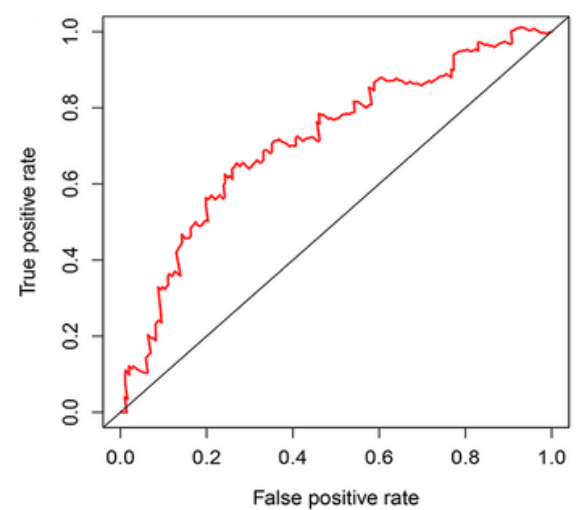

$\mathrm{h}$.

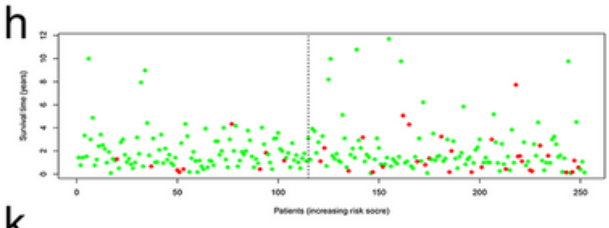

k

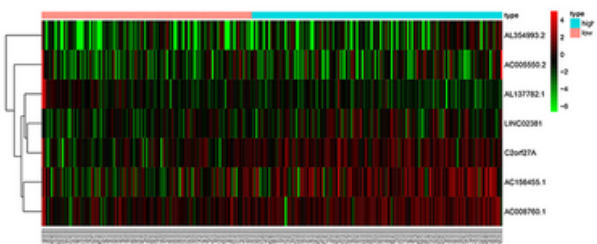

Entire set

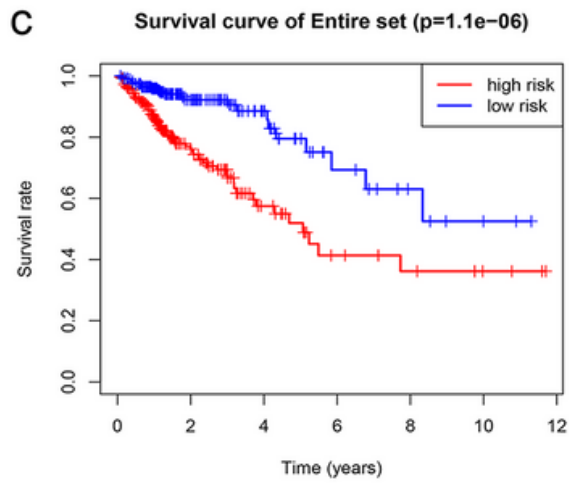

f
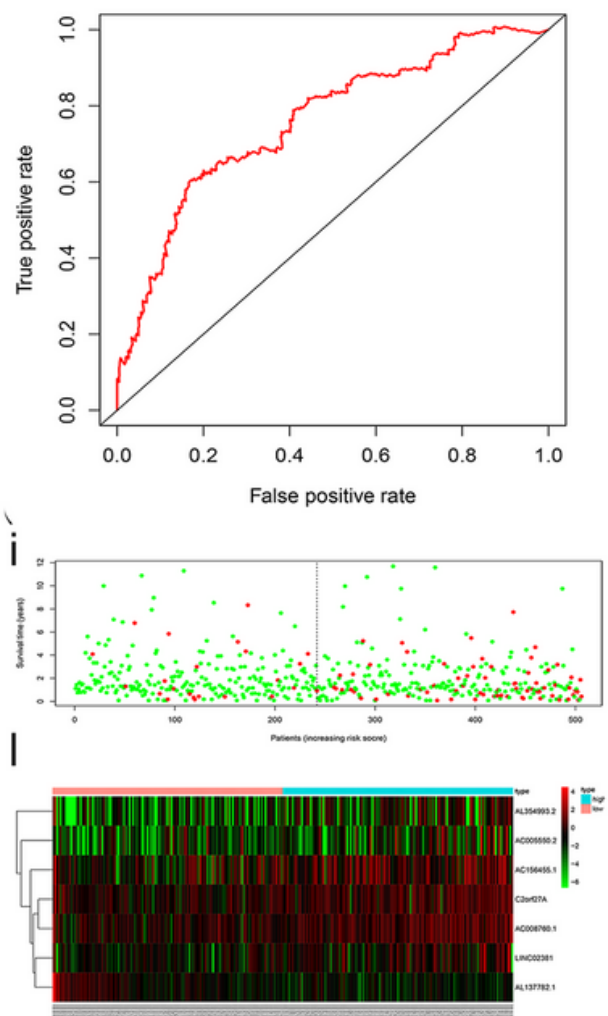

\section{Figure 2}

Validation and evaluation of the ferroptosis-related IncRNAs prognostic signature. Kaplan-Meier curves in the train set (a), test set (b), entire set (c); The AUC of three years dependent curve in the train set (d), test set (e), entire set (f); Survival status in high and low-risk patients for train set (g), test set (h), entire set (i), red dots represent death, and green dots represent alive; The cluster heat map of seven IncRNAs' expression in high and low risk groups for the train set (j), test set (k), entire set (l) 

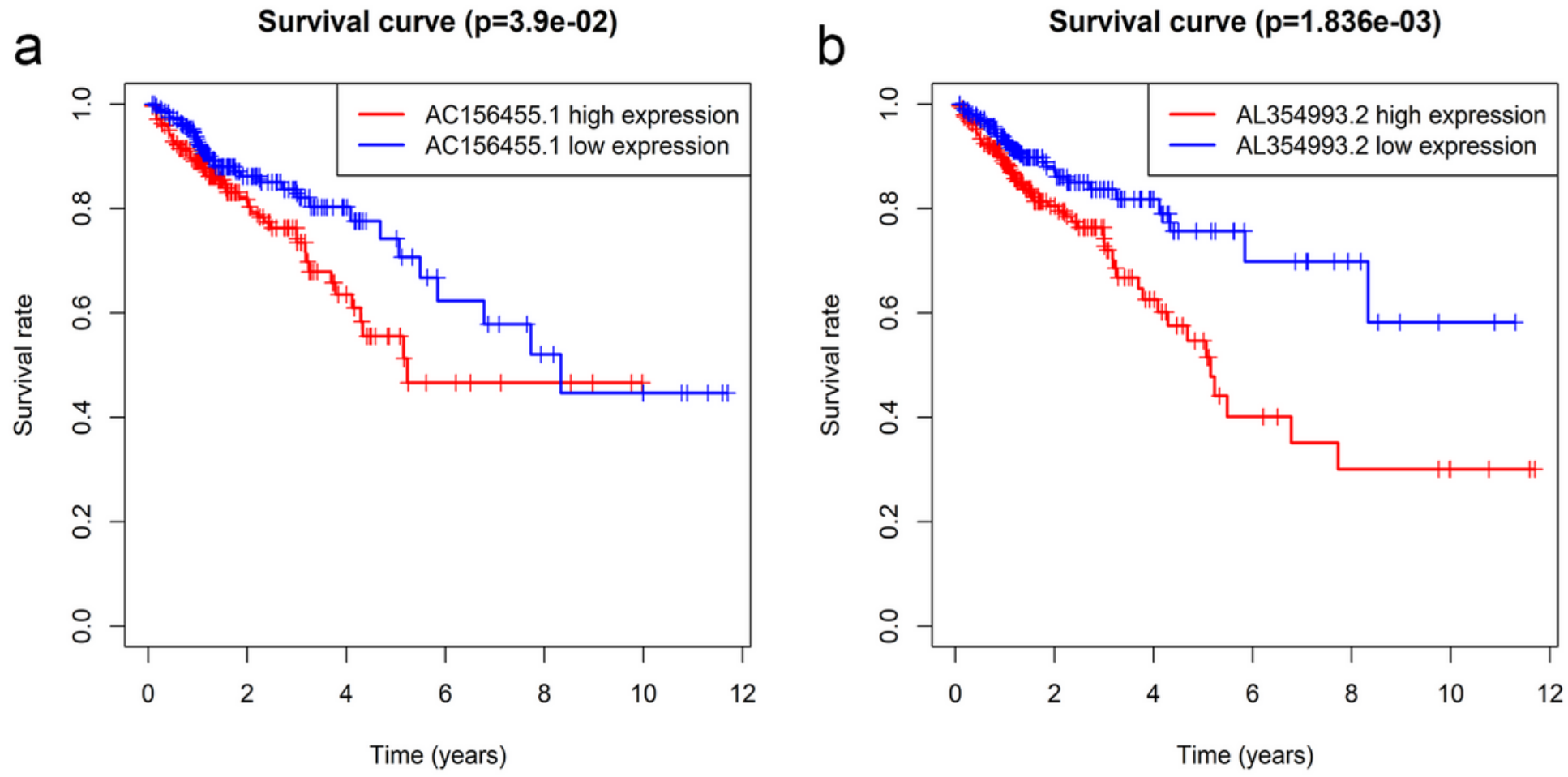

Figure 3

Two IncRNAs associated with overall survival in CRC patients using Kaplan-Meier curves and log-rank tests. The patients were stratified into high and low expression groups based on the median expression of each IncRNA. a AC026355.1. b AC099850.3. 


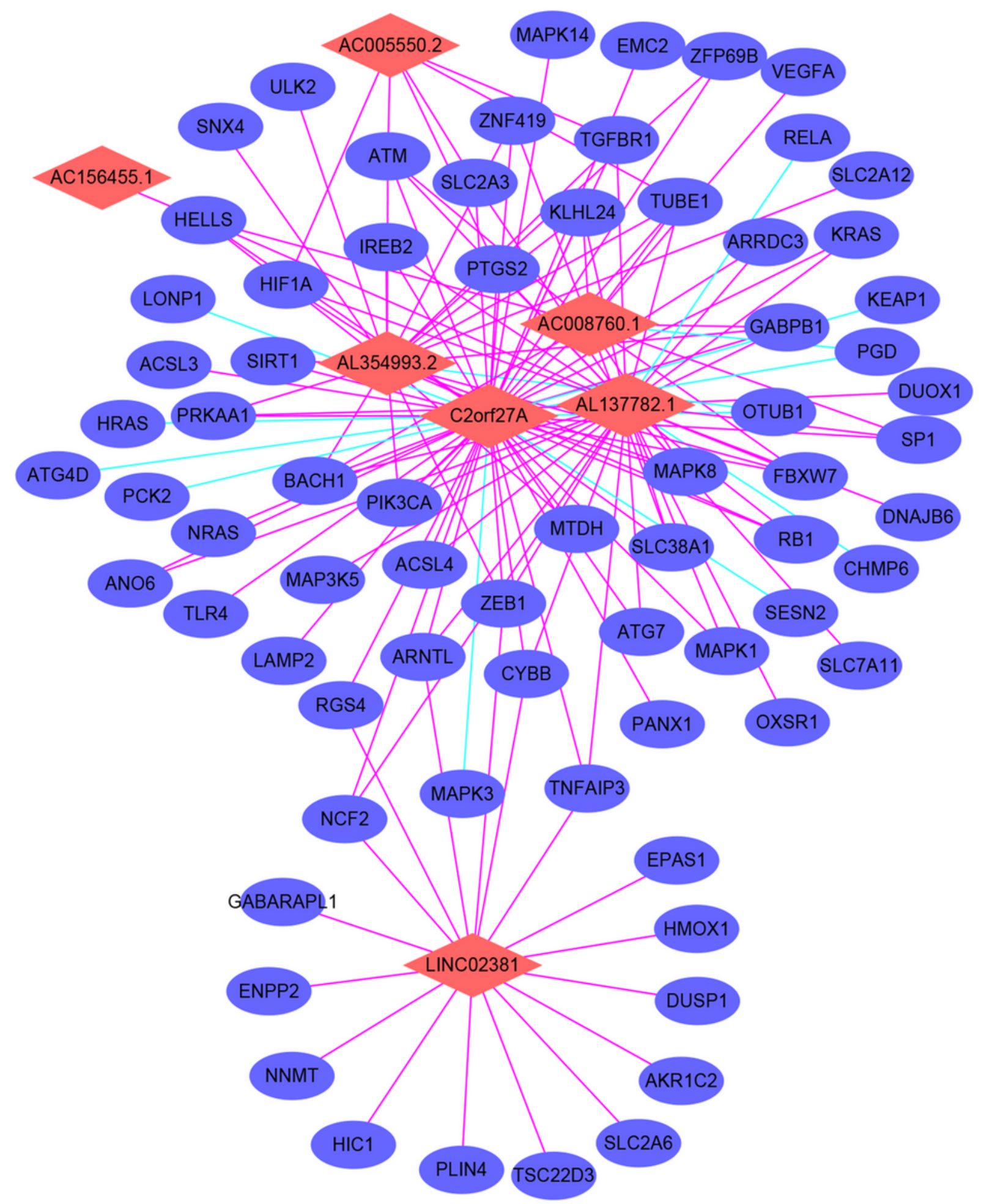

\section{Figure 4}

The network relationship between the seven IncRNAs and ferroptosis genes. Prismatic represent IncRNA, and oval represent ferroptosis genes. The red line represents positive correlation, and blue represents negative correlation. 

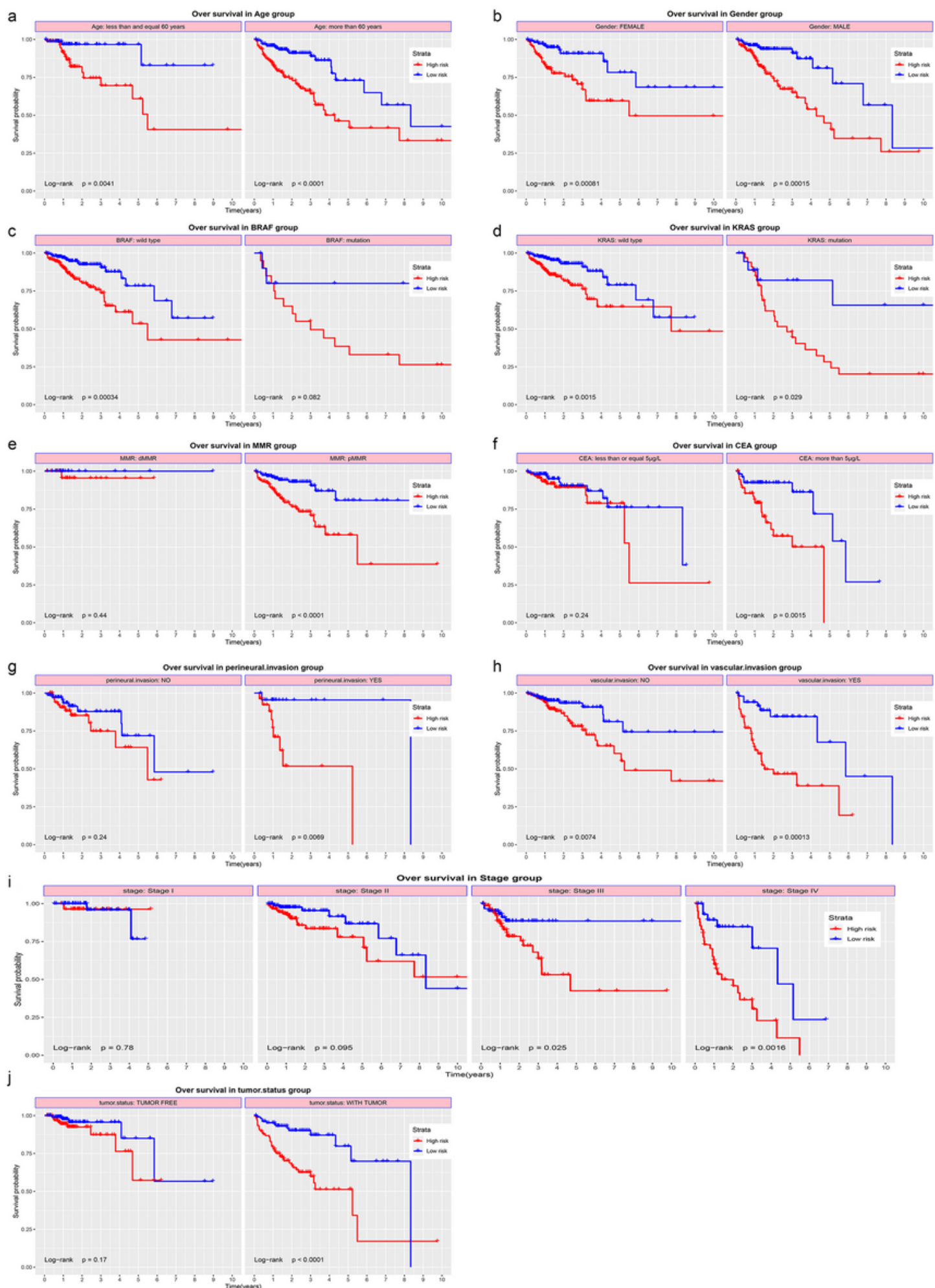

Figure 5

The overall survival of the ferroptosis-related IncRNAs prognostic signature in the stratification of clinical variables. a Age group. b Gender group. c BRAF group. d KRAS group. e MMR group. f CEA group. $g$ Perineural invasion group. $\mathrm{h}$ Vascular invasion group. i Stage group. $\mathrm{j}$ Tumor status group. 


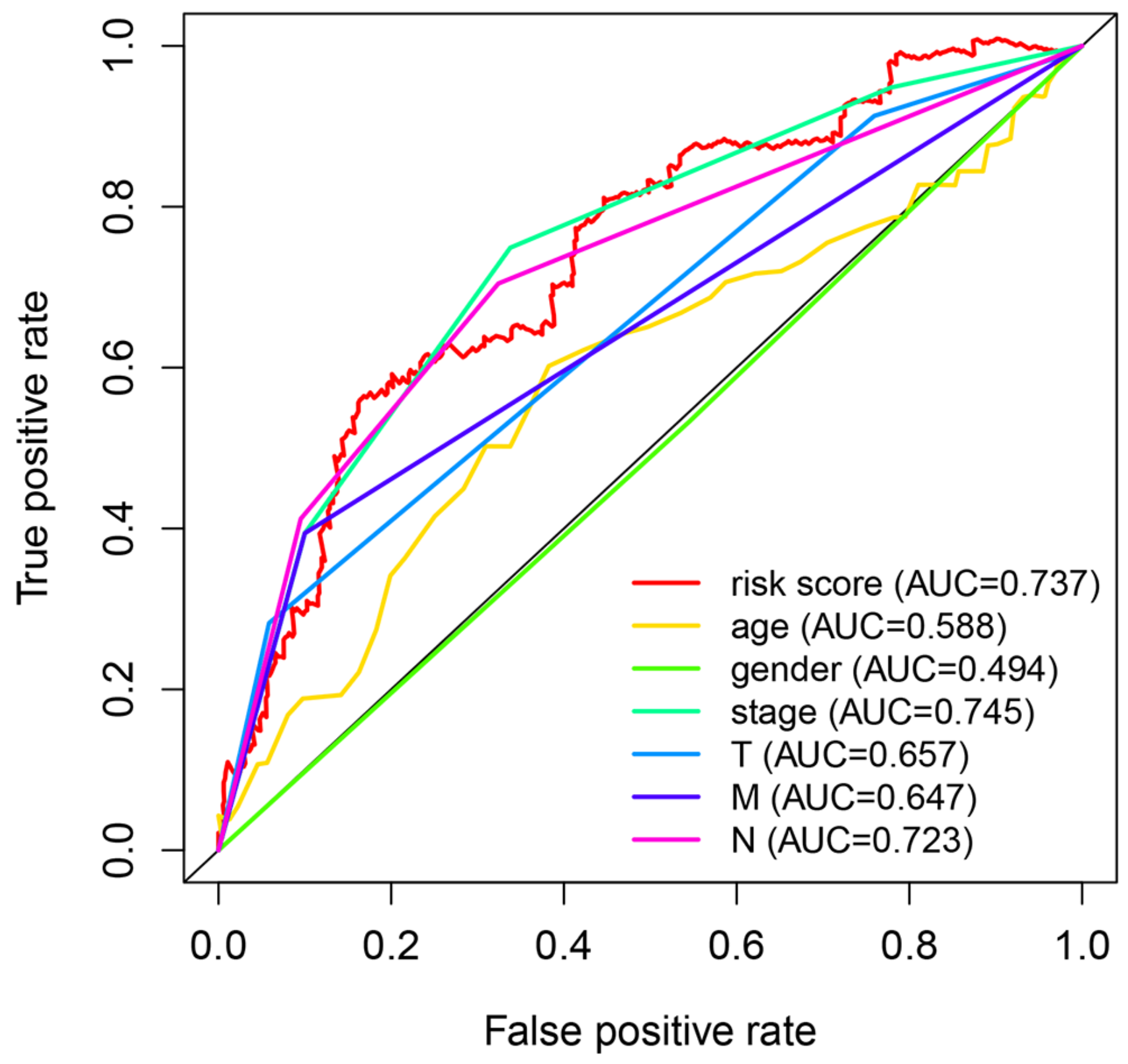

Figure 6

The ROC curve of the ferroptosis-related IncRNAs prognostic signature and clinical variables. 

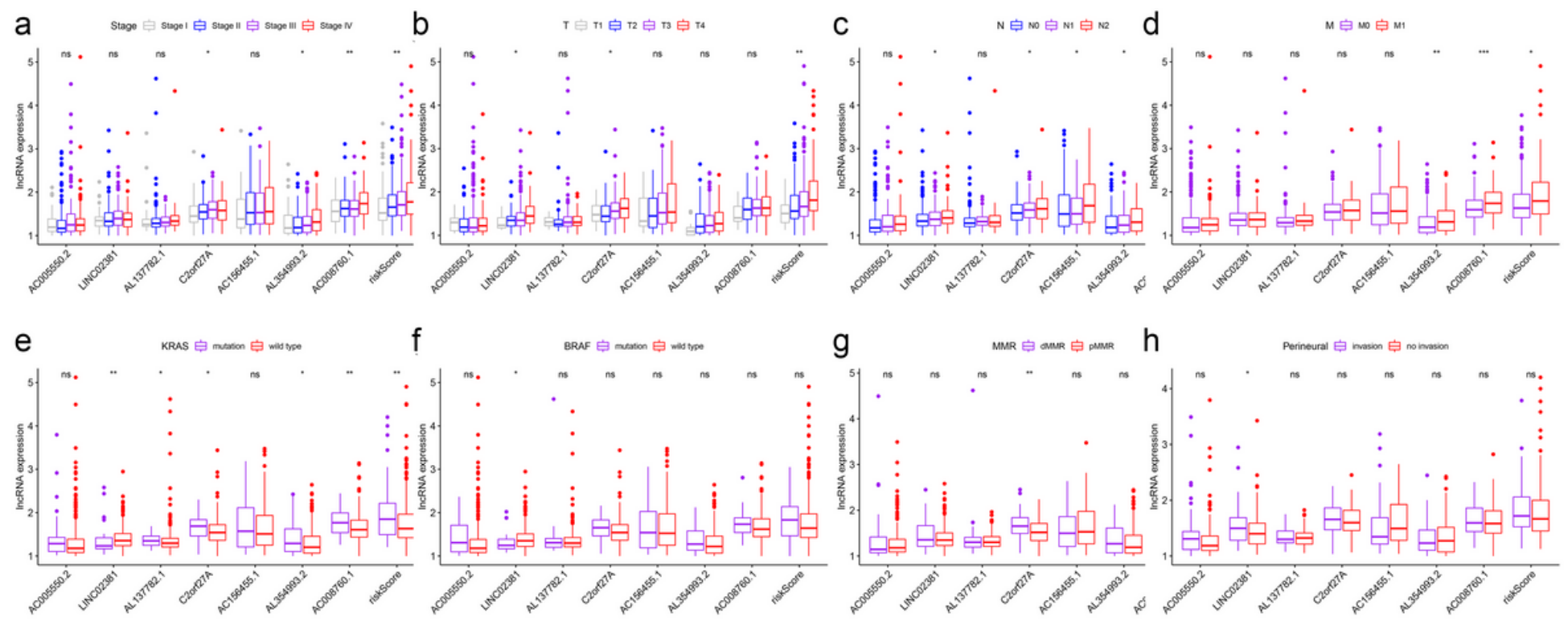

\section{Figure 7}

The correlation between the IncRNAs constituting the signature and clinical variables. a Clinical stage. $b$ T stage. c Lymph-node status. d Distant metastasis. e KRAS status. f BRAF status. g MMR. h Perineural invasion. * represents $P<0.05$, ** represents $P<0.01$, *** represents $P<0.001$, "ns" represents no statistical significance.

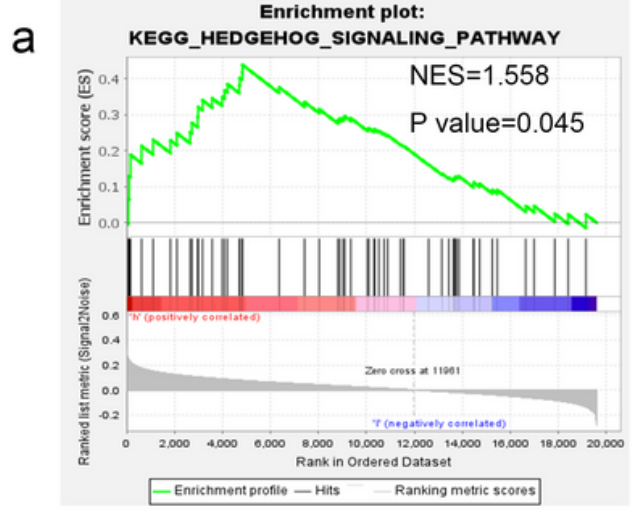

d

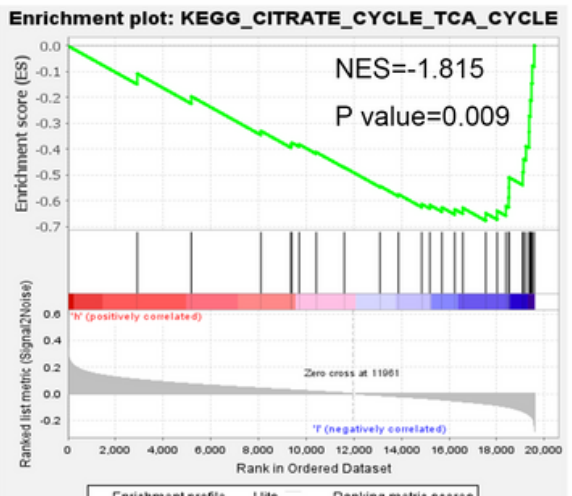

b
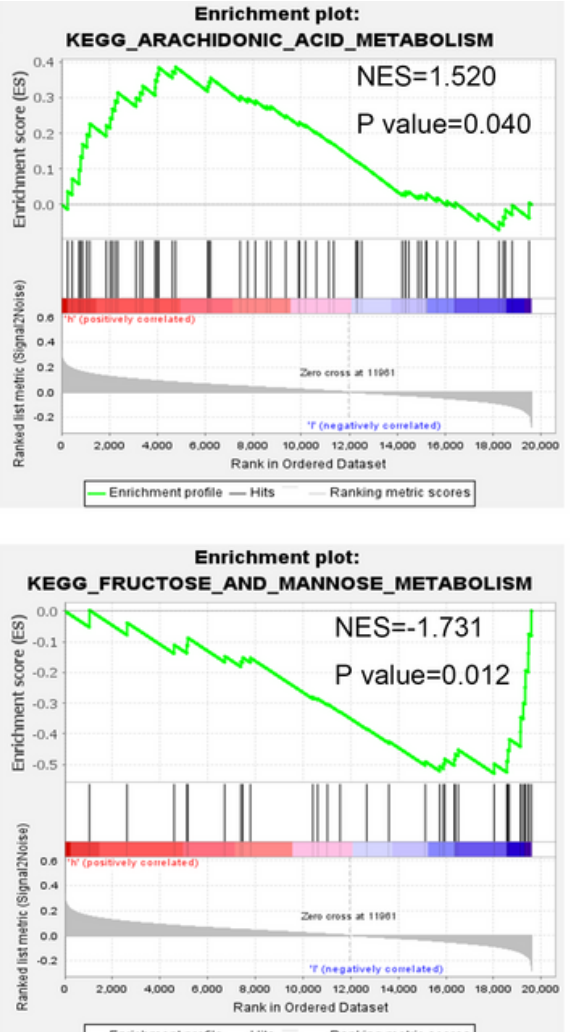

Enrichment plot: C KEGG_ALPHA_LINOLENIC_ACID_METABOLISM

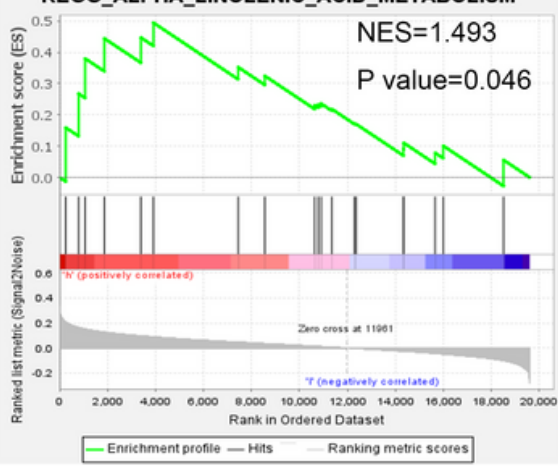

f

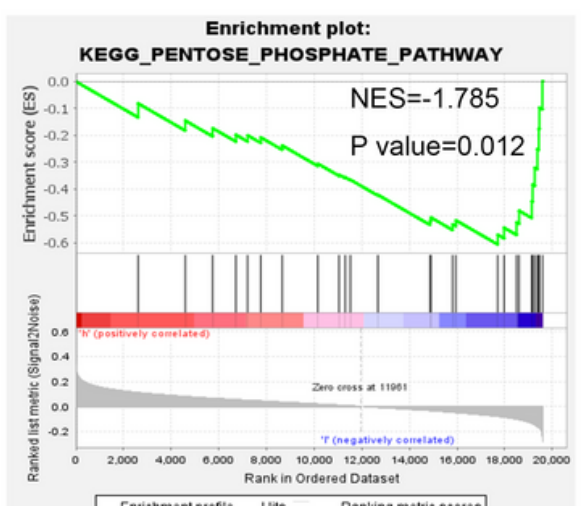

Figure 8 
The KEGG enrichment analysis of GSEA enriched in the high-risk group and low-risk group of the ferroptosis-related IncRNAs prognostic signature. a kegg hedgehog signaling pathway. b kegg arachidonic acid metabolism. c kegg alpha linolenic acid metabolism. $d$ kegg citrate cycle tca cycle. e kegg fructose and mannose metabolism. f kegg pentose phosphate pathway.

\section{Supplementary Files}

This is a list of supplementary files associated with this preprint. Click to download.

- Table1.xlsx

- Table2.xlsx

- Additionalfile1.xlsx

- Additionalfile2.xlsx

- Additionalfile3.xlsx

- Additionalfile4.xIsx

- Additionalfile5.xlsx

- Additionalfile6.xlsx

- Additionalfile7.xlsx

- Additionalfile8.xlsx

- Additionalfile9.xlsx 